\title{
Further Discussion of Binary Star Radio Survey Data
}

\author{
E. Budding ${ }^{1,2}$, O. B. Slee ${ }^{3}$ and K. Jones ${ }^{4}$ \\ ${ }^{1}$ Central Institute of Technology, PO Box 40740, Upper Hutt, Wellington, New Zealand \\ ${ }^{2}$ Carter Observatory, PO Box 2909, Wellington, New Zealand \\ Edwin.Budding@cit.ac.nz \\ ${ }^{3}$ Australia Telescope National Facility, PO Box 76, Epping, NSW 2121, Australia \\ bslee@atnf.csiro.au \\ ${ }^{4}$ Physics Department, University of Queensland, St Lucia, Qld 4072, Australia \\ jones@physics.uq.oz.au
}

Received 1997 May 19, accepted 1998 March 18

\begin{abstract}
Statistical information on 8280 individual radio observations of binary stars, predominantly at $8.4 \mathrm{GHz}$ using the Parkes $64 \mathrm{~m}$ antenna, is presented. Three main groups are distinguished: (i) RS CVn stars, (ii) classical Algol binaries (EA2s), and (iii) detached pairs of generally early type (ETBs).

The RS CVn stars more frequently gave rise to detectable fluxes, while the ETBs, in these data, are a small and rather heterogeneous class. The Algols' emission appears to increase near conjunction phases, though we cannot clearly distinguish any special property of the Algols' phase-dependent behaviour that is not also shared by the RS CVn binaries. Both these categories' data show a bimodal, phase-dependent pattern to the distribution of detections, suggesting that these binary types share similar underlying physical properties, though there could also be other factors at play. The sample sizes of the Algols and particularly the ETB detections are too small for effective, discriminatory statistics, however.
\end{abstract}

Keywords: binary stars - RS CVns-Algols—radio emission

\section{Introduction}

Slee et al. (1987) have published data from microwave (predominantly $8.4 \mathrm{GHz}$ ) surveys of binary stars carried out at Parkes in the eighties. The present article revisits and extends the data on systems observed at Parkes, generally between mid-1981 and late 1989, with a few points added from more recent ATCA observations.

The stars concentrated on here are those for which $3 \sigma$ detections were reported. They have been assigned to three groups as follows: (i) $16 \mathrm{RS} \mathrm{CVn}$ stars (AR Psc, AT Cap, BH Ind, CF Tuc, EI Eri, GT Msc, HD101309, IL Hya, IM Peg, IN Vel, RZ Eri, SZ Psc, TW Lep, V1379 Aql, V711 Tau \& V824 Ara) (cf. Strassmeier et al. 1993); (ii) 8 classical Algol binaries ( $\delta$ Lib, KZ Pav, R CMa, RY Gem, S Cnc, V393 Sco, V505 Sgr \& XZ Sgr) (EA2s, cf. Budding 1985); and (iii) 6 early-type detached pairs ( $\delta$ Cap, TZ Men, V760 Sco, V822 Aql, V1647 Sgr \& $\zeta$ Phe) (ETBs, cf. Stewart et al. 1988). In the survey of Slee et al. (1987) there were 48,25 and 18 stars altogether in these groups, respectively. All the binaries studied hereafter have known photometric ephemerides.

The original data samples consist of source intensity measurements over background measured at a specified offset position ('wags'), together with standard errors of the individual determinations. Precautions in procedure designed to eliminate the effects of possible source confusion were reported previously by Slee et al. (1987). The average value of this standard error over the whole data collection is a little over $2 \mathrm{mJy}$. There are 828 entries in our total subset, each a mean of 10 individual measurements, and $3 \sigma$, or greater, detections occur in about $41 \%$ of these entries. The most frequently detected sources are the RS CVn binaries, which account for almost $75 \%$ of the measurements, some of which (particularly V711 Tau = HR1099) are very frequently observable. In fact, both RS CVn stars and Algol binaries are well known radio emitters (cf. e.g. Linksy 1994). Although the significance of early reported detections in certain other subsections of the Parkes stellar surveys (Slee et al. 1989) has been called into question (Beasley et al. 1992), on the basis of standard deviations some $20 \%$ lower than we apply here, the emissions we shall discuss carry internal evidence against spurious or accidental registration. Further informed discussion of the determination of appropriate significance measures has been presented by Slee \& Budding (1995).

All the observed sources have an irregular character to the emission, and the majority must be detected when an outburst is taking them, sporadically, 

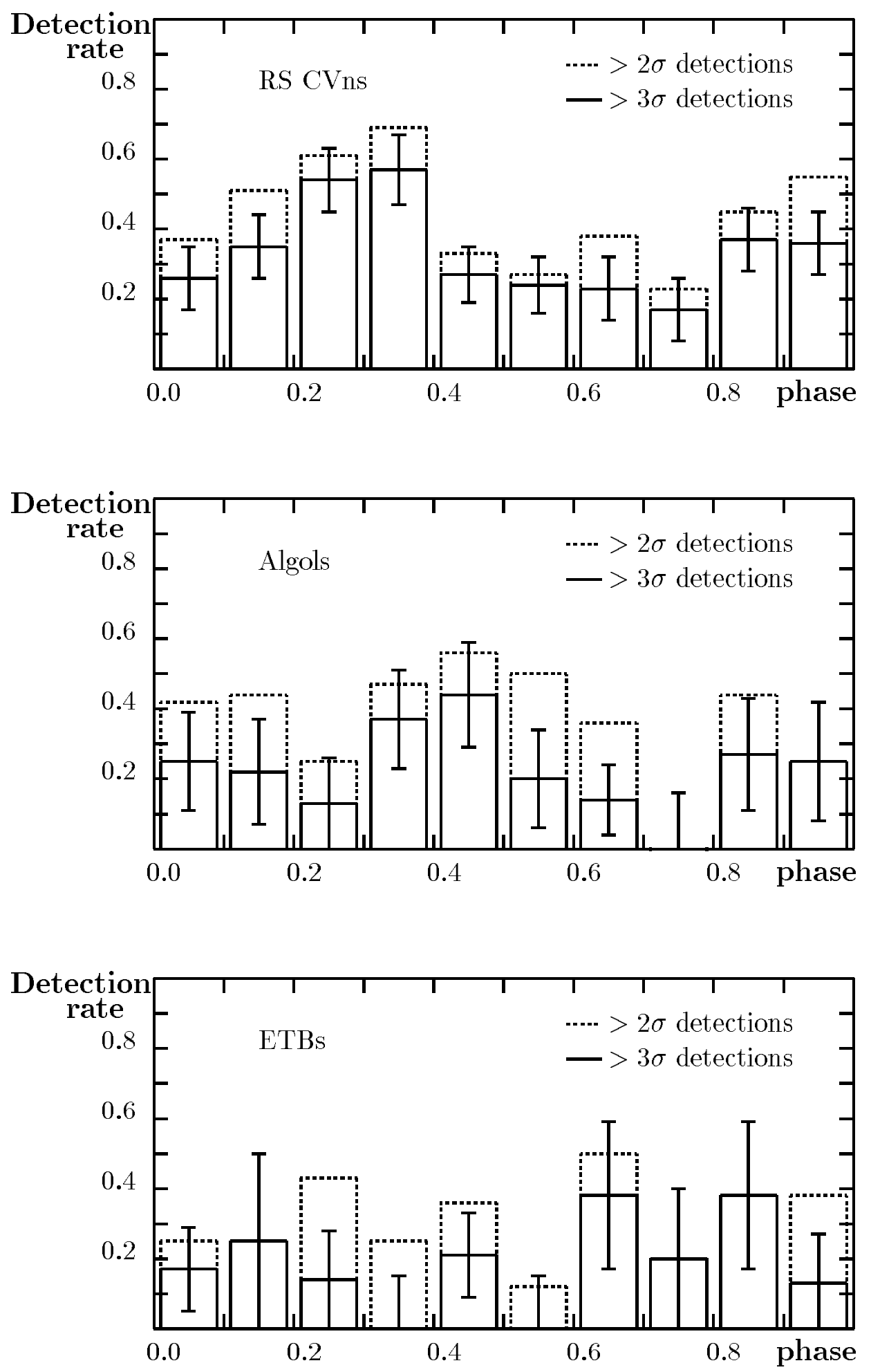

Figure 1-Distribution in phase of detections of RS CVn stars (top), classical Algols (middle), and early type pairs (bottom).

above detection threshold. An inverse-square law in distance would affect the threshold received power, but the evidence shows other factors to be also strongly at play in determining detection incidence. A (heliocentric) binary phase can be determined for each radio measurement, so one clear purpose is to look for any orbital phase dependence in such detections.

\section{Results}

We show, in the histograms of Figure 1, the distributions in phase of $>2 \sigma$ and $>3 \sigma$ rates of detection for the RS CVn, EA2 and ETB groups. These are formed by dividing the number of registered detections by the total number of observation entries in each $0 \cdot 1$ interval of phase. The phase sampling 
Table 1. Summary of $3 \sigma$ detection statistics for RS CVn and Algol binaries

Shown are combined observations and detections, average detection incidence per bin for the whole set suitably 'normalised' to the number of observations in each phase bin, detection rate (detections/observations), probable (Poissonian) errors and $\chi^{2}$ deviations from a uniform incidence. The $\chi^{2}$ values are derived by squaring the difference of the two numbers in the 7th and 8th columns, and dividing by the latter entry

\begin{tabular}{|c|c|c|c|c|c|c|c|c|c|c|}
\hline \multirow{2}{*}{$\begin{array}{l}\text { Phase } \\
\text { bin }\end{array}$} & \multicolumn{3}{|c|}{ Observations } & \multicolumn{3}{|c|}{$3 \sigma$ detections } & \multirow{2}{*}{$\begin{array}{c}\text { Normalised } \\
\text { incidence }\end{array}$} & \multirow{2}{*}{$\begin{array}{l}\text { Detection } \\
\text { rate }\end{array}$} & \multirow[t]{2}{*}{ Error } & \multirow[t]{2}{*}{$\chi^{2}$} \\
\hline & RS's & Al's & Sum & RS's & Al's & Sum & & & & \\
\hline $0 \cdot 05$ & 35 & 12 & 47 & 9 & 3 & 12 & $15 \cdot 6$ & $0 \cdot 255$ & $0 \cdot 073$ & $0 \cdot 83$ \\
\hline $0 \cdot 15$ & 49 & 9 & 58 & 17 & 2 & 19 & $19 \cdot 3$ & $0 \cdot 328$ & $0 \cdot 075$ & $0 \cdot 00$ \\
\hline $0 \cdot 25$ & 57 & 8 & 65 & 31 & 1 & 32 & $21 \cdot 6$ & $0 \cdot 492$ & $0 \cdot 087$ & $5 \cdot 01$ \\
\hline $0 \cdot 35$ & 49 & 19 & 68 & 28 & 7 & 35 & $22 \cdot 6$ & 0.515 & $0 \cdot 087$ & $6 \cdot 80$ \\
\hline $0 \cdot 45$ & 48 & 18 & 66 & 13 & 8 & 21 & $21 \cdot 9$ & $0 \cdot 318$ & $0 \cdot 069$ & $0 \cdot 03$ \\
\hline 0.55 & 45 & 10 & 55 & 11 & 2 & 13 & $18 \cdot 3$ & $0 \cdot 236$ & $0 \cdot 066$ & 1.53 \\
\hline $0 \cdot 65$ & 39 & 14 & 53 & 9 & 2 & 11 & $17 \cdot 6$ & $0 \cdot 208$ & $0 \cdot 063$ & $2 \cdot 78$ \\
\hline $0 \cdot 75$ & 35 & 6 & 41 & 6 & 0 & 6 & $13 \cdot 6$ & $0 \cdot 146$ & $0 \cdot 060$ & $4 \cdot 24$ \\
\hline $0 \cdot 85$ & 49 & 11 & 60 & 18 & 3 & 21 & $19 \cdot 9$ & $0 \cdot 350$ & $0 \cdot 076$ & $0 \cdot 06$ \\
\hline \multirow[t]{2}{*}{$0 \cdot 95$} & 42 & 8 & 50 & 15 & 2 & 17 & $16 \cdot 6$ & $0 \cdot 340$ & $0 \cdot 082$ & $0 \cdot 01$ \\
\hline & & & & & & & Av. $18 \cdot 7$ & & & $\Sigma 20 \cdot 99$ \\
\hline
\end{tabular}

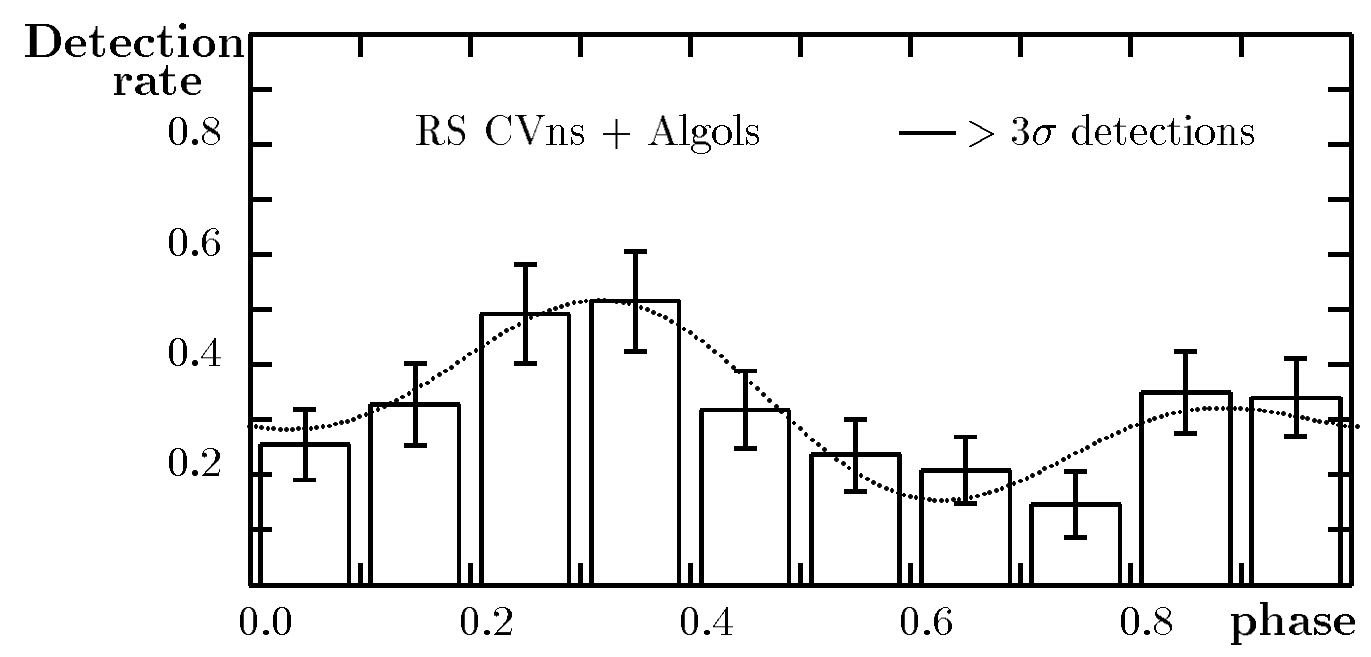

Figure 2-Distribution in phase of detections of RS CVn stars and Algols.

is fairly uniform in these phase bins for the binary groups studied.

Easiest to deal with, perhaps, is the ETB group, which is consistent with no particular phase preference for detections, although the sample is too small for reliable statistics (only $15>3 \sigma$ detections).

The detections of the RS CVn stars also appear to be without very strong phase linking, although there is more variation than goes with a purely random distribution. If we include V711 Tau, $\chi^{2}$ sums of the $3 \sigma$ detection rates, compared with the mean rate $(0.44)$ for the whole set, indicate a probability of about $21 \%$ that the observed phase distribution could correspond to a random example of one that is intrinsically uniform. However, V711 Tau is detected essentially at all phases. If this diluting effect is removed, the phase distribution of the remaining, more distant RS CVn detections becomes distinctly less uniform. This distribution is illustrated in Figure 1 (top). (V711 Tau is considered later in Figure 3.) The probability of the distribution of Figure 1 (top) being a random example from a uniform source drops to about
$1 \cdot 3 \%$, according to the sum of its deviations from the mean, tested with the $\chi^{2}$ function for 9 degrees of freedom (cf. e.g. Conover 1980).

The outburst detection rate's variation with phase $r(\phi)$, shown in Figure 1 (top), is adequately modelled, for a Poisson distribution of deviations from the model's predictions, by a three element Fourier series, of the form (phase shifts in degrees)*

$$
\begin{aligned}
r(\phi)= & 0 \cdot 336+0 \cdot 122 \cos (\phi+280)-0 \cdot 099 \cos 2(\phi-29) \\
& \pm 0 \cdot 021 \pm 0 \cdot 030 \quad \pm 14 \quad \pm 0 \cdot 030 \quad \pm 8 \cdot 6 .
\end{aligned}
$$

The detection rates for the Algol systems in Figure 1 also show some departure from uniform behaviour, however, the small numbers in the data set imply correspondingly large error bars. A $\chi^{2}$ test indicates only a little more than even chance

${ }^{*}$ The \pm error limits given below equations (1)-(3) correspond, respectively, to each numerical parameter on the right hand side. 
(54\%) of a non-uniform parent distribution for this sample. The observed EA2 detections' trend is well represented by

$$
\begin{aligned}
r(\phi)= & 0 \cdot 227+0 \cdot 076 \cos (\phi+248)+0 \cdot 126 \cos 2(\phi+20) \\
& \pm 0 \cdot 108 \pm 0 \cdot 15 \quad \pm 11 \quad \pm 0 \cdot 15 \quad \pm 34,
\end{aligned}
$$

which is effectively within its broad errors of the formula used for the RS CVn stars.

The RS CVn and Algol binaries were explored as a single class by combining their detection statistics. Relevant data are summarised in Table 1. Results are shown diagrammatically in Figure 2. This distribution of $3 \sigma$ detections of the combined groups satisfies

$$
\begin{array}{r}
r(\phi)=0 \cdot 323+0 \cdot 12 \cos (\phi+271)-0 \cdot 090 \cos 2(\phi-33) \\
\quad \pm 0 \cdot 018 \pm 0 \cdot 026 \pm 12 \quad \pm 0 \cdot 026 \quad \pm 8 \cdot 2 .
\end{array}
$$

There is an improvement in the quality of the three-term Fourier representation over equation (1), and $\chi^{2}$ testing indicates a probability of just about $1 \%$ that this data sample is drawn from a uniform parent distribution. This two-harmonic Fourier representation is shown as the dotted curve in Figure 2. We can thus reasonably infer that the observed phase distribution of the rates of microwave detections for Algol and RS CVn binaries is consistent with their sharing certain key underlying physical similarities bearing on the flux generation.

The most effective Algol performer in the sample ( $\delta$ Lib, detected in $75 \%$ of observations) appears to accentuate the heterogeneity of emission with phase. All 13 flux measurements above $12 \mathrm{mJy}$ are within 0.16 of phases 0.45 and $0.95 ; 92 \%$ are within $45^{\circ}$ and $62 \%$ are within $35^{\circ}$ of these phases. The sampling rate of the 28 observations is not too uniform, however. Even so, there are five observations in both the $0 \cdot 3-0 \cdot 4$ and $0 \cdot 4-0 \cdot 5$ phase ranges, but the accumulated flux in the latter bin is four times that of the former.

There are generally much more frequent measurable fluxes of RS CVn stars than the EA2 or ETB groups. Thus V711 Tau gives a $>3 \sigma$ detection in $98 \%$ of cases, while $\delta$ Cap, at only $1 / 3$ of the distance $(\sim 12 \mathrm{pc})$, achieves this in only $\sim 1 / 6$ of its observations. Slee et al. (1987) showed, however, that the increased detection rate of RS CVn stars is influenced by their greater spatial incidence than that of classical Algols. The median brightness temperatures of Algols were found to be several times higher than those of RS CVn stars by Slee et al. (1987), while the median distance of that sample of Algols was about $50 \%$ greater. There is, however, considerable overlap in the emission characteristics of the two groups, the Algols' surface fluxes falling within the range of that of the RS CVn stars, though it should also be noted that $4 \cdot 6$ times as many observations of RS CVn stars as Algols were made altogether.

The four eclipsing RS CVn stars (V 1379 Aql, RZ Eri, SZ Psc and CF Tuc) have a slightly smaller median surface flux than the remaining 12 noneclipsing ones, but their mean $3 \sigma$ detection rates are essentially the same $[0 \cdot 33 \pm 0 \cdot 06$ for the eclipsers, as against $0 \cdot 35 \pm 0 \cdot 03$ (excluding V711 Tau) for the noneclipsers]. The mean distances and distance ranges of these two groups are sufficiently comparable as to indicate that there is no effective way of statistically discriminating between eclipsing and non-eclipsing RS CVn binary emission effects in our data. The very frequent detections of the low-inclination $\left(33^{\circ}\right) \mathrm{RS}$ CVn binary V711 Tau might favour polar visibility as a factor in detectability, but this is not verified on the basis of any simple statistical correlation with flux levels and non-eclipses. The radio-bright and relatively near V711 Tau shows occasionally very large and apparently randomly distributed emissions superposed over what appears as a more steady background. This background variation also suggests a phase dependency, maximising towards phases $\sim 0.4$ and $\sim 0.9$ (Figure 3 ).

By averaging the full detection rates of the RS $\mathrm{CVn}$ stars, including now the non-detections for the remaining 32 stars of Slee et al. (1987), we estimate that for a typical RS CVn star at a distance of about one hundred pc, about one observation in three would result in a positive detection with the set-up used in the Parkes survey. The mean distance of the $16 \mathrm{RS} \mathrm{CVn}$ stars studied in this paper is $155 \mathrm{pc}$. There is a great variation in the absolute detectabilities of different systems, however. For example, the well-known eclipsing binary $\mathrm{CF}$ Tuc (HIPPARCOS distance $86 \mathrm{pc}$ ) was detected in about one in four observations, as against one in seven for IN Vel, which is six times more distant. Indeed, the detections seem to include some selection effects which reflect a disproportionate influence of unusually active remote RS CVn stars.

Proceeding similarly, we find the $3 \sigma$ detection expectancy of a classical Algol at a hundred pc to be about $0 \cdot 2$. The 8 Algols in this study have an average distance some 1.3 times greater than that of the RS CVn systems, though the overall range of distances of EA2 stars is comparable to that of the RS CVns (a few tens to several hundreds of pc). It is worth noting that the Algol $3 \sigma$ detection probability, in our data, is enhanced by a factor of $1 \cdot 8$ in the phase ranges $0 \cdot 4-0 \cdot 5$ and $0 \cdot 9-1 \cdot 0$ over the remainder of the phase cycle.

Referring back again, briefly, to the ETB detections: the rate is very heterogeneous in this small selection. For example, the relatively near $\delta$ Cap 

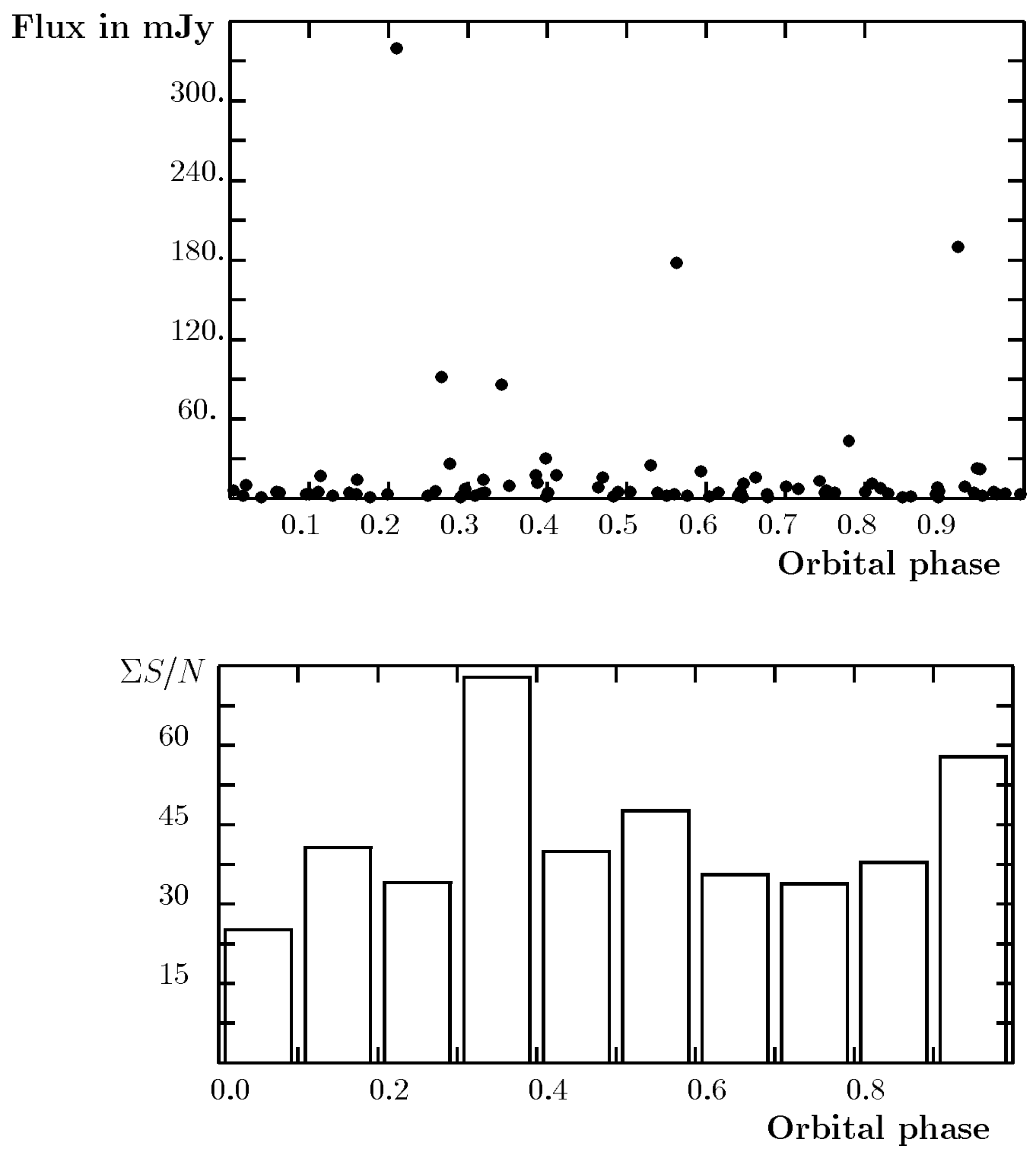

Figure 3-(upper) Distribution of flux measurements with phase for V711 Tau (HR 1099); (lower) distribution of accumulated relative $S / N$ values for V711 Tau. The six highest values have been removed to show any trend to the 'background' emission.

has a $3 \sigma$ detection incidence of $0 \cdot 17$, while V822 Aql, at some $680 \mathrm{pc}$ distance, has a $3 \sigma$ detection rate of $0 \cdot 22$. The latter binary has a very early-type (B3) primary, and is photometrically classified as an EB (strong tidal distortions) binary (Kholopov 1985), despite its $5 \cdot 3$ day period. It may then well resemble $\beta$ Lyrae, or a massive Algol at the early stages of its interaction history. Overall, the detection rate for the ETBs appears to be about half that of the classical Algols.

\section{Discussion}

The indication of a non-uniform, bimodal tendency in the phase distribution of detectable emissions from certain binary classes raises interest in the physics of their configurations. RS CVn stars are a well known source of microwave radiation, and there are many corroborative factors that link this radiation with enhanced magnetodynamic stellar 'activity' (cf. e.g. Linsky 1988, 1994). The $3 \sigma$ detection distribution and the background radiation trend for V711 Tau suggest some preferred longitudes for the visibility of magnetodynamic phenomena. This is in keeping with 'active longitude' sector concepts, which have been frequently discussed. With surface fields of order several thousand gauss concentrated over regions of order $10^{8} \mathrm{~m}$ in radius, magnetic reconnection events can be expected to energise electrons at least to tens of MeV. Such electrons would subsequently lose energy by synchrotron radiation in the confining fields of the active region, and may be the source of the detected outbursts (Dulk 1985). It would thus be a useful exercise to carry out a more detailed inter-comparison of radio emission and photometric properties of both RS CVn and Algol systems (Budding 1996). 
From another point of view, the long-term, quasicontinuous and relatively substantial flow of matter from a 'loser' to a 'gainer' characterises Algols, or other stars undergoing Roche lobe overflow (RLOF). The phase range $0 \cdot 4-0 \cdot 5$, where our Algols' detections maximise, corresponds to where the mass-transferring plasma stream would be seen coming towards the observer over the limb of the primary star. The possibility of a signature of the RLOF stream in the microwave range has only been briefly considered previously (e.g. in Slee et al. 1987), as far as we know. It is suggested, however, by the discussion of the location of origin for the microwave emission from polars like V834 Cen, which also indicate a phase-linked pattern to their emission (cf. Chanmugam \& Dulk 1982; Wright et al. 1988; Ferrario Wickramasinghe \& Tuohy 1989). So, insofar as certain gross features of the mass-transferring stream's microwave emission in polars may resemble that of Algols, we might expect some comparability of observed effects.

It could also be interesting to examine this idea further. In considering any potentially relevant properties of the mass-transferring stream specific to Algols, reference can be made to Lubow \& Shu's (1975) classical Algol model. This allows for kinetic energy transport in the transferred material typically of the order of $10^{-2}$ of the gainer luminosity (cf. e.g. Plavec 1989), or up to say, $\sim 10^{27} \mathrm{~W}$, for the classical Algols observed. If we estimate field strengths near the gainer-stream interaction region to be of order 10 gauss, where the hypersonic stream velocities attain $\sim 10^{-3} \mathrm{c}$, the Faraday induction across the stream could easily be of order $0 \cdot 1 \mathrm{~V} \mathrm{~m}^{-1}$, developing emfs of around $10^{7} \mathrm{~V}$ across an interaction ('hot spot') region of lateral extent $10^{8} \mathrm{~m}$. The detections of the Algols at the mJy level require that up to $10^{21} \mathrm{~W}$ be emitted at source over the whole microwave spectrum during the outbursts. The sporadically occurring emissions are most frequently observed when the induced electic field would be predominantly perpendicular to the line of sight, if the relevant region of the gainer's magnetic field is essentially poloidal and nearly perpendicular to the by passing stream. Since outbursts are observed only $\sim 10 \%$ of the time, we require $\sim 10^{-7}$ of the available steady kinetic power. This kinetic power is of the same order as the Poynting energy developed in the hypothesised interaction region with the foregoing parameter values.

At present, it has to be admitted that the Parkes evidence for any special effects in Algol systems is suggestive only. A significantly higher median brightness temperature of Algols over RS CVn stars was noted earlier (Slee et al. 1987), but we have not been able effectively to distinguish any difference in phase-dependent characterstics by comparing our Algol and RS CVn binary microwave detection statistics. Although it would be interesting to know if there was any signature of the characteristic stream of Algol binaries at radio wavelengths, a more sensitive survey than the Parkes one would be required to find out. In the meantime it appears more pragmatic to regard the processes giving rise to Algol and RS CVn binary radio emissions as essentially similar.

\section{Acknowledgments}

E. Budding acknowledges support from the New Zealand side of the Bilateral Research Agreement Programme (BRAP) of the Ministry of Research, Science \& Technology (NZ), 1996, to enable visits to the Australia Telescope National Facility, where the ideas for this paper were mooted. We also acknowledge the initial unpublished analysis of Dr $\mathrm{K}$. Thompson of Monash University on the subject of orbital dependency in the Parkes stellar observations.

\section{References}

Beasley, A. J., Stewart, R. T., \& Carter, B. D. 1992, MNRAS, 254, 1

Budding, E. 1985, Proc. 3rd IAU Asian Pacific Regional Meeting, ed. M. Kitamura \& E. Budding (Dordrecht: Kluwer), p. 241

Budding, E. 1996, in Stellar Surface Structure, IAU Symp. 176, ed. K. G. Strassmeier \& J. L. Linsky (Dordrecht: Kluwer), p. 95

Chanmugam, D., \& Dulk, G. A. 1982, ApJ, 255, L107

Conover, W. J. 1980, Practical Nonparametric Statistics (New York: Wiley)

Dulk, G. A. 1985, ARA\&A, 23, 169

Ferrario, L., Wickramasinghe, D. T., \& Tuohy, I. R. 1989, ApJ, 341, 327

Kholopov, P. N. 1985, General Catalogue of Variable Stars (Moscow: Nauka)

Linsky, J. L. 1988, in Multiwavelength Astrophysics, ed. F. A. Cordova (Cambridge Univ. Press), p. 49

Linsky, J. L. 1994, in Solar Coronal Structures, IAU Colloq. 144, ed. V. Rusin et al. (Bratislava: Veda), p. 1

Lubow, S. H., \& Shu, F. H. 1975, ApJ, 198, 383

Plavec, M. J. 1989, Space Sci. Rev., 50, 95

Slee, O. B., Nelson, G. J., Stewart, R. T., Wright, A. E., Innis, J. L., Ryan, S. G., \& Vaughan, A. E. 1987, MNRAS, 229, 659

Stewart, R. T., Innis, J. L., Slee, O. B., Nelson, G. J., \& Wright, A. E. 1988, AJ, 96, 371

Strassmeier, K. G., Hall, D. S., Fekel, F. C., \& Scheck, M. 1993, A\&AS, 100, 173

Wright, A. E., Cropper, M., Stewart, R. T., Nelson, G. J., \& Slee, O. B. 1988, MNRAS, 231, 319 